\title{
EDITORIAL
}

\section{THE NURSING HOME POPULATION: AN OPPORTUNITY TO MAKE ADVANCES ON RESEARCH ON MULTIMORBIDITY AND POLYPHARMACY}

\author{
P. DE SOUTO BARRETO', B. VELLAS ${ }^{1,2}$, J.E. MORLEY ${ }^{3}$, Y. ROLLAND $^{1,2}$
}

\begin{abstract}
1. Gérontopôle de Toulouse, Institut du Vieillissement, Centre Hospitalo-Universitaire de Toulouse (CHU Toulouse), Toulouse; 2. Inserm U1027, F-31073, Avenue Jules Guesde, University of Toulouse III, F-31073, France; 3. Division of Geriatric Medicine, Saint Louis University School of Medicine, 1402 S. Grand Blvd., M238, St. Louis, Missouri USA. Corresponding author: Dr. Philipe de Souto Barreto. Gérontopôle de Toulouse, Institut du Vieillissement. 37, Allées Jules Guesde. 31000 Toulouse, France, Telephone number: (+33) 561145 664, Fax: (+33) 561145 640, e-mail: philipebarreto81@yahoo.com.br
\end{abstract}

Multimorbidity constitutes a challenge for health care professionals. However, as indicated in a report from the US Institute of Medicine (1), evidence-based research on the clinical management of multimorbid patients remains scarce, especially for people 85 years or over (2). This is even more true for multimorbid patients living in nursing homes $(\mathrm{NH})(3)$. Current clinical practices in multimorbid patients living in NHs represent a crude translation from research in other populations (e.g., hospitalized patients, community-dwelling older adults) (4). Yet, NH residents constitute a particular population, with specific care needs (5). Moreover, their health status is partially dependent on $\mathrm{NH}$ leadership and internal organization (3). In this Editorial, we discuss the clinical importance and methodological advantages of performing research specifically in $\mathrm{NH}$ residents, and the role of the primary care physician for the development of successful clinical research among residents.

There is an urgent need to perform high quality research among multimorbid NH residents. Indeed, based on exclusion criteria (including multimorbidity, and institutionalization itself), these NH residents are frequently excluded from randomized controlled trials (RCT), especially pharmacological RCTs $(3,4)$. However, multimorbid residents $(6)$, with a high rate of adverse health events and frequently polymedicated, would more particularly benefit from clinical research designed to improve health outcomes and demonstrate the value of a specific treatment. That is why a group of experts in geriatrics, on the behalf of the International Association of Gerontology and Geriatrics and The World Health Organization, have recently urged that "national drug approval agencies consider requiring drug trials that are age appropriate and inclusive of nursing home residents before they are approved" (3).

NHs should be regarded as a methodologically attractive research setting. First of all, interventions being developed in the life space of the study participants, research monitoring may be facilitated and compliance to the intervention improved. Moreover, close monitoring, which is feasible in NHs, may improve adherence rates to intervention and follow-up. This is all the more important as dropout rates in RCTs performed in older adults are high because they usually necessitate long follow-ups as exemplified by trials in the field of dementia (7); this also may probably be the case for research on multimorbidity. Furthermore, information on the reasons of dropout may be more reliable as well as easily available in $\mathrm{NH}$ residents (e.g., using medical charts, institutional records, and the MDS 3.0) (8) than in community-dwellers. Finally, the availability of data specific to $\mathrm{NH}$ residents may generate highly reliable results on the efficacy and safety of new interventions for the frail elderly.

Evidence-based research on multimorbidity among $\mathrm{NH}$ residents strongly relies on the participation of primary care physicians, who, in many countries, continue to prescribe multiple drugs even after people are admitted to a $\mathrm{NH}$. However, primary care physicians are frequently confronted by the problem of lack of time (9), which may constitute an important obstacle to their involvement in clinical research. This difficulty may be even greater for research in $\mathrm{NH}$ residents, as it may necessitate physicians having to visit his/her patient to the NH. Alternatively, residents may come to the medical office, but they frequently have important functional limitations (5), making this extremely difficult. In this context, collaborative models of care may prove to be more adequate to the complex needs of people with multimorbidity (10). Nevertheless, organizing multidisciplinary teams (MDT) is not simple. We are currently testing MDT feasibility and effectiveness in a RCT $(n=1428)$ for the detection of Alzheimer's disease among NH residents (11). Preliminary results of the first MDT (held in $32 \mathrm{NHs}$ ) found a low participation rate of primary care physicians (4 among the 133 health professionals who attended to the MDT meetings). Whilst using telehealth for promoting MDT may constitute an attractive solution to increase physicians' participation, its feasibility and effectiveness needs to be proven. A community of practice model for MDT has been similarly utilized in Scotland and a preliminary study in NHs has suggested it can reduce falls in NHs (12).

Overall, there is an urgent need to perform high quality research among NH residents (2). This population represents a methodologically attractive population for research on multimorbidity and polypharmacy. In this context, new practical approaches allowing high rates of participation of primary care physicians in the research development in the $\mathrm{NH}$ are needed. Involving these professionals in the study design phase could facilitate research development in NHs. The 


\section{RESEARCH ON MULTIMORBIDITY IN NURSING HOMES}

American Medical Directors Associatoin (AMDA) Foundation has piloted this approach, and while finding it difficult, has shown it is feasible $(13,14)$. In Toulouse we have been successfull in involving community physicians in research (15) and have developed a nursing home network (16-19) which should allow for intervention studies.

Acknowledgments: We thank Dr Yves Cadroy (MD - Gérontopôle, CHU Toulouse) for assisting with the English editing; he did not receive compensation for his work. work.

Conflict of interest: The authors declare no conflict of interest.

Data accessibility: All authors had access to the data and a role in writing the manuscript.

\section{References}

1. Institute of Medicine. Living well with chronic illness: a call for public health action. National Academies Press, 2012.

2. Taekema DG, Gussekloo J, Westendorp RGJ, de Craen AJM, Maier AB. Predicting Survival in Oldest Old People. Am. J. Med. 2012; In Press.

3. Tolson D, Rolland Y, Andrieu S, Aquino J-P, Beard J, Benetos A, et al. International Association of Gerontology and Geriatrics: a global agenda for clinical research and quality of care in nursing homes. J Am Med Dir Assoc. 2011;12(3):184-9.

4. Rolland Y, Barreto PS. Research can improve care in the nursing home. J Am Med Dir Assoc (In Press).

5. Barreto PS, Lapeyre-Mestre M, Mathieu C, et al. A multicentric individually-tailored controlled trial of education and professional support to nursing home staff: research protocol and baseline data of the IQUARE study. J Nutr Health Aging 2013;17(2):173-178

6. Field TS, Tjia J, Mazor KM, Donovan JL, Kanaan AO, Harrold LR, et al Randomized trial of a warfarin communication protocol for nursing homes: an SBAR-based approach. Am. J. Med. 2011;124(2):179.e1-7
7. Andrieu S, Coley N, Aisen P, Carrillo MC, DeKosky S, Durga J, et al. Methodological issues in primary prevention trials for neurodegenerative dementia. $\mathrm{J}$ Alzheimers Dis. 2009;16(2):235-70.

8. Saliba D, Jones M, Streim J, et al. Overrview of significant changes in the Minimum Data Set for nursing homes version 3.0. J Am Med Dir Assoc 2012;13:595-601.

9. Kadam U. Redesigning the general practice consultation to improve care for patients with multimorbidity. BMJ. 2012;345:e6202

10. Boult C, Wieland GD. Comprehensive primary care for older patients with multiple chronic conditions: « Nobody rushes you through ». JAMA. 2010;304(17):1936-43.

11. Rolland Y, Tavassoli N, Gillette-Guyonnet S, et al. Multidisciplinary Team Meetings (MDTM) in Detection of Alzheimer's Disease: Data from the IDEM Study. J Nutr Health Aging 2013;17(2):137-141.

12. Tolson D, Lowndes A, Booth $\mathrm{J}$, et al. The potential of communities of practice to promote evidence-informed practice within nursing homes. J Am Med Dir Assoc 2011;12:169-173.

13. Stange K, Libow L. AMDA Foundation research network. J Am Med Dir Assoc 2002;3:330-336.

14. Dharmarajan TS, Nanda A, Agarwal B, et al. Prevention of venous thromboembolism: practice patterns in 17 geographically diverse long term care facilities in the United States, part 1 of 2 (an AMDA Foundation project). J Am Med Dir Assoc 2012;13:298-302

15. Subra J, Gillette-Guyonnet S, Cesari M, Oustric S, Vellas B; Platform Team.The integration of frailty into clinical practice: preliminary results from the Gérontopôle.J Nutr Health Aging. 2012 ;16(8):714-20. doi: 10.1007/s12603-012-0391-7.

16. de Souto Barreto P, Lapeyre-Mestre M, Mathieu C, Piau C, Bouget C, Cayla F, Vellas B, Rolland Y. Prevalence and Associations of the Use of Proton-Pump Inhibitors in Nursing Homes: A Cross-Sectional Study. J Am Med Dir Assoc. 2012 Dec 1. doi:pii: S1525-8610(12)00407-0. 10.1016/j.jamda.2012.10.018. [Epub ahead of print] PubMed PMID: 23211534.

17. de Souto Barreto P, Lapeyre-Mestre M, Mathieu C, Piau C, Bouget C, Cayla F, Vellas B, Rolland Y. Indicators of benzodiazepine use in nursing home residents in france: a cross-sectional study. J Am Med Dir Assoc. 2013 Jan;14(1):29-33. doi: 10.1016/j.jamda.2012.09.002. Epub 2012 Oct 23. PubMed PMID: 23098415.

18. Rolland Y, Perrin A, Gardette V, Filhol N, Vellas B. Screening older people at risk of malnutrition or malnourished using the Simplified Nutritional Appetite Questionnaire (SNAQ): a comparison with the Mini-Nutritional Assessment (MNA) tool. J Am Med Dir Assoc. 2012 Jan;13(1):31-4. doi: 10.1016/j.jamda.2011.05.003.

19. Rolland Y, Abellan van Kan G, Hermabessiere S, Gerard S, Guyonnet Gillette S, Vellas B. Descriptive study of nursing home residents from the REHPA network.J Nutr Health Aging. 2009;13(8):679-83. 